\title{
An account of liver-related clinical data maintained in a single hepatopancreaticobiliary surgical unit using a cost-free user-friendly database management solution
}

\author{
Malaka JAYAWARDENE*, Malith Sachintha NANDASENA, Upul DE SILVA, Aloka PATHIRANA
}

Department of Hepatopancreaticobiliary Surgery, Professorial Surgical Unit, Colombo South Teaching Hospital, Sri Lanka

Introduction: Maintaining robust clinical data in a low-income country is a challenge due to the lack of electronic medical record systems in state sector hospitals. To mitigate this, the author created a cloud-based cost-free app for the HPB unit of Colombo South Teaching Hospital.

Methods: Analyzable data variables pertaining to HPB patients were prospectively gathered over a period of 25 months using the app. The app's on-the-go analysis section was used to extract liver-related data.

Results: $36 \%(n=566)$ of the HPB data were liver-related. $66 \%$ were males. Mean-age was $65.75 \%$ of liver lesions were malignancies; 57\% $(\mathrm{n}=242)$ HCC, 16\% $(\mathrm{n}=66)$ colorectal liver metastasis (CRLM), 5\% $(\mathrm{n}=21)$ non-colorectal liver metastasis (NCRLM). 45\% of HCC belonged to 61 to 70 age group. Male:female of HCC 4.8:1, CRLM 1.2:1, and NCRLM 0.9:1. 51\% of HCC were solitary-lesions, $30 \%$ multiple-lesions. $25 \%$ of CRLM were solitary-lesions, $43 \%$ multiple-lesions. $53 \%$ of HCC were confined to right-lobe, $31 \%$ to leftlobe. CRLM $58 \%$ in right-lobe, $37 \%$ in both lobes. $64 \%$ of HCC were discrete-HCC, $30 \%$ were diffuse-HCC. Of those with conclusive decisions, $38 \%(n=130)$ of liver-lesions surgically, and $62 \%(n=214)$ non-surgically were managed. Lesions were resectable in $31 \%$ of HCC, $74 \%$ of CRLM, and $80 \%$ of NCRLM. $43 \%(\mathrm{n}=12)$ of resectable-HCC underwent hemihepatectomy, $36 \%(\mathrm{n}=10)$ NALR, $11 \%$ trisectionectomy. For CRLM, this was 60\% $(n=15)$ NALR, 36\% $(n=9)$ hemihepatectomy. 54\% $(n=58)$ of inoperable-HCC had locoregional-therapy; $19 \%$-ablation (72\%-thermal, 28\%-chemical) and $81 \%$-TACE. $46 \%$ of inoperable-HCC were palliated.

Conclusions: This form of mobile-based clinical data maintenance has improved the data completion rates and accuracy of HPB data. 